\title{
Role of Difficulty in Emotion Regulation, Suicidal Ideation and Distress in Patients with Substance use disorders
}

\begin{abstract}
Muhammad Zakaria, Shazia Hasan
Department of psychology, University of Central Punjab,Pakistan

Abstract- Cognitive theory explains that the substance intoxication develops a syndrome which directly affects the central nerves system. It further creates troubles in perception, thinking, concentration and emotion regulation. The difficulty in emotion regulation problem causes the suicidal ideation and psychological distress in substance patients, but most of the clinician is unaware about the role of emotion dysregulation in substance intoxication and other psychological problems that they didn't provided specific treatment for emotion regulation problem to controlled substance intoxication and its related problems. The present study explore the role of difficulty in emotion regulation with suicidal ideation and psychological distress among patients with substance use disorders. The date was collected from 60 patients with substance use disorders, with age range from 18 to 35 years. The sample size was arranged through the G Power software. Data of the current study was collected from different government and private hospitals and drug rehabilitation centers of Lahore city Pakistan by using of convenient sampling technique. The difficulty emotional regulation, suicidal ideation attributes and depressions, Anxiety, Stress (DASS) scales were used for data collection. The simple linear regression statistical analysis was used for data analysis. Simple linear regression analysis indicated that difficulty in emotion regulation $(\beta=.214)$ is positively associated with suicidal ideation and psychological distress, depression is $(\beta=.322)$, anxiety is $(\beta=.326)$ and stress $(\beta=.320)$. Among patients with substance use disorders. Difficulty in emotion regulation has positively predicated the suicidal ideation and psychological distress among patients with substance use disorders. The present study was found that the difficulty in emotion regulation plays a significant role in suicidal ideation and psychological distress among patients with substance use disorders.
\end{abstract}

Keywords: Difficulty in emotion regulation, Psychology distress, Suicidal ideation

Copyright (c) 2020: Muhammad Zakaria. This is an open access distribution, and reproduction in any medium, provided Access article distributed under the Creative Commons Attribution License the original work is properly cited License, which permits unrestricted use.

Citation: Muhammad Zakaria. "Role of Difficulty in Emotion Regulation, Suicidal Ideation and Distress in Patients with Substance use disorders, 1(3), 61-66, July-August 2020.

\section{INTRODUCTION}

Different substance used by human beings is not a new experience but the scientific classification and diagnosis of substance use disorders only begin in the early 19th century.1The ancient people were used the different plants as a remedy in pain, but at the time of modernization and industrialization the use of substance increased severely and cases the different severe psychological and environmental problems. People use to consume such substances as food which cause extreme dependency and change the system of the body and brain function. Equally, when these substances are abused they modify mental and physical functions of normal human. The different substance are divided into different groups such as depressants, like Opium, morphine, cannabis, methadone, and other narcotics and stimulants, Hallucinogens, (Cocaine, and Peyote, other hallucinogens); and these substances are frequently used in all over the world. 2
In last $\quad 35$ years the substance addiction model has changed two times in Pakistan. In 1983 there were only hardly five thousands substance abusers. In 1987 the second change of substance addicts' model were observed when the cannabis was introduced in 1980s. During short time of five years, the number of substance abuse increased from 20000 , to 365,000 . In 1985 , there were two million cannabis addicts in Pakistan. The ratio increase to 3.1 million abusers in 1993.The 2002 survey reported that the cannabis was the most accepted substance used by 2.1 million people around. The ratio of substance users rise to 4.1 million which is $(2.8 \%)$ of the overall population, 1 million substance user are increased in only 8 years. Now the substance abuse ratio has increase to 8 million. 3

Substance abuse is an abnormal way of substance use which leads to clinically major impairment or psychological problems in which the individual may also experience 
phases of tolerance and withdrawal.4 the difficulty in emotion regulation appears to be a significant contributor of etiology and maintenance of substance abuse. The difficulty in emotion regulation included both positive and negative affect as well as effortful control that are associated to substance abuse. The difficulty in emotion regulation not only describes the connection of initiation and risk for substance abuse, but also connected to the way of developmental stages of substance abuse. 5 Researches and literature review revealed that difficulty in emotion regulation motivates/initiates a person to use and become greater barriers in quitting the substance; the difficulty in emotion regulation has also shown that the victims are motivated to frequently use of cannabis.6 similarly, the difficulty in emotion regulation was also found in heroin abusers.7

Cognitive theory explains that the substance intoxication develop a syndrome which directly affect the central nerves system. It further creates troubles in perception, thinking, concentration and decision making abilities. Self-regulation is a significant factor caused by substance use disorders. Self-regulation or emotion regulation includes execution of action plan to change the way of behaviors. 8 Changing of circumstances in self-regulation develop the imbalance and poor ability to control the emotions. Addictive behaviors are seen as the consequence of having an extreme dependence on external structures that have less ability to regulate emotion properly. Substance dependent individuals excessively use substance to overcome psychological problems, but the over use of substance increase the psychological problems and may also causes the suicidal thoughts and attempts 9

Number of substances directly affects the brain and causes psychological distress, e.g., cannabis slow down brain functioning and affect the cognitive ability dimension and causes distress. According to Beck (1976), 11 negative thoughts, generate by core thinking are usually the major cause of psychological distress. A direct connection occurs among the severity of negative thoughts and severity of their distress.11, 12The psychological interpersonal model of suicide indicated that the relationship among difficulty in emotion regulation and suicidal behaviors. The model explore that the suicidal risk is partially function of required ability to involve in self-injury and this ability may occur through hurting and challenging experience.13 Another study supporting this model to establish the association among difficulty in emotion regulation and required skill for suicide attempts connecting to courage about self-hurt and pain acceptance was mediated by the experience of painful and provoking life events.13 The escape theory of suicidal behavior is helpful in explanation of relationship of difficulty in emotion regulation and suicidal ideation 14 the theory further explains that people consider suicide as an option when they are incapable to produce alternative to getaway situation that they find to be emotionally unbearable. Some painful emotions may happen difficult if person has lack of essential ability to control their emotions. 15

\section{II.LITRATRURE REVIEW}

Bender et al., (2011) study indicate that difficulty emotion regulation is associated with suicidal behavior among substance user disorders. The different aspects of difficulty in emotion regulation notable between people with a record of several suicidal attempts, a single attempt, in substance use disorders individuals.

A research result show that the difficulty in emotion regulation is the tendency to react to a negative mood and causes anxiety, depression and other disorders over the time to predict suicidal ideation in substance abuse.13 Several theories of suicide and substance abuse have indicated that the relationship of substance abuse and suicide exists. The theories briefly indicated that the individuals with depress mood are more prone to use the self-medicate substances. Otherwise, the substance abuse is correlated to high levels of aggression and impulsivity that turn to conduct problems that can support risk for suicidal behaviors. e.g., sometimes the significant relationship of substance abuse and suicide were decreased when other risky behaviors are present, e.g., stealing, gambling, sexual behaviors. 14

The research result indicated that significant relationship among psychological distress (depression, anxiety and stress) and substance abuse. The depression and substance abuse are highly co-morbid in major two condition, they occur together in a high percentage among adults. In some situation depression cause the drug addiction and sometime drug addiction can cause depression. Number of substances use directly affects the brain function and cause psychological distress. E.g., cannabis slows down brain functioning and affect the cognitive abilities and cause distress. Many people with depression control themselves by self-medications. When they're depressed, they have difficulty in accepting that they 'treated the depression by medications or psychotherapy but many people turn to medication for solutions. 16

Different researches literature show the significant relationship among psychological distress and substance abuse in adults. The distress and substance abuse are highly co-morbid in major two condition, they occur together in a high percentage among adults. In some situation distress cause the drug addiction and sometime drug addiction can cause psychological distress. 16

The research results indicates the relationship among mood disorders and drug addiction, those individuals who were diagnose with a mood disorder are probable to drug addiction than a individual without mood disorders.17 Different research studies indicated that the substance abuse increases the severity level of depressive symptoms.18

Kelly (2004) conducted the research study to examine the correlation between psychological distress and substance use disorders. Substance abuse histories and information 
were received. To assess distress with substance use disorders, the anxiety and depression and stress. The study result revealed that $46.1 \%$ substance abuse had psychological distress.

\section{A. Significances of the present study}

The purpose of study is to investigate the emotion dysregulation, suicidal ideation and psychological distress (depression, anxiety, stress) among patients with substance use disorders and non-users. This study would be focusing on finding that how emotion dysregulation leads towards drug addiction and when emotions are regulated, may that have positive effects on people. The finding of this research is assist in developing an insight among clinicians, researchers and policy makers regarding drug abusers psychological functioning and developing laws, rules and regulation for providing medication and properly working on drug abusers' health for controlling suicides and other risk factors.

\section{B. Hypotheses}

Hypotheses of the present study are as follows.

1. Difficulty in emotion regulation would be positively predicted the suicidal ideation and psychological distress (depression, anxiety and stress) among patients with substance use disorders.

2. Difficulty in emotion regulation has positively predicated the suicidal ideation and among patients with substance use disorders.

\section{III.RESEARCH METHODS}

\section{A. Research design and Participants}

The cross section and correlation research design was used in current research study. . The date was collected from 60 patients with substance use disorders, with age range from 18 to 35 years. The sample size was arranged through the G Power software. Age range of the participants was selected based on previous studies which shown that the young age individuals have more emotion problems which lead to negative coping skills and psychological issues. Data was collected from different government and private hospitals and drug rehabilitation centers of Lahore city Pakistan by using of convenient sampling technique.

\section{B. Measurement Tools}

Demographic form was used to collect the all demographic information such as age, gender, education, relapse, socioeconomic status and marital status etc. Difficulty in Emotional Regulation Scale (DERS) by Gratz \& Romer (2004) was used to assess Difficulty in Emotional Regulation of participants.19. Suicidal Ideation Attributes Scale (SIAS) by Spijker et al., (2014) was used to measure the suicidal ideation of participants. 20

Depression Anxiety and Stress Scale (DASS) by Lovibond \& Lovibond (1995) was used to assess the Depression Anxiety and Stress of participants.21.

\section{Inclusion}

1. The participants with Relapses in the sample were included.

2. The duration of substance use from 1 to 3 years were included.

3. The participants living with family were included.

4. The participants with no history of mental disorder and physical handicap were included.

5. Substance withdrawal participants were included.

\section{Exclusion}

3. The participants with criminal history were excluded.

4. The drug intoxication participants were excluded.

5. The participants with multiple substance use history were excluded.

Data Analysis: The Statistical Package for the Social Sciences (SPSS version 21) was used to analyze the date. The descriptive statistics was used to describe the characteristics of the participants. The Simple Linear Regression was used to analyze the hypothesis of the study.

Procedure: Initially permission was obtained from the authorities regarding data collection. Then, participant was brief about the purpose of the research study. They were assured about the confidentiality of information obtained from them. Immediate counseling and therapeutic recommendation would was given to participants.

\section{F. Limitations}

Limitation of this study concerns the use of self- report tools and measures to assess the difficulty in emotion regulation, suicidal ideation and psychological distress (depression, anxiety and stress) among patient with substance use disorders and non-users. Our study comprised on the sample was taken from the rehabilitation centers. We can gather more accurate results, if the data were collected from different cities rehabilitation centers

\section{RESULTS}

The current chapter included Simple Linear Regression analysis. The following chapter provides the detailed analysis of data which was collected from patient with substance use disorders. Simple Linear Regression analysis was conducted to examine the relationship among difficulty in emotion regulation, suicidal ideation and psychological distress among patients with substance use disorders.

The results of present research reveal that difficulty in emotion regulation is positively predicted the suicidal ideation $(\beta=.214)$ among patients with substance use disorders. The results seen in (Table 1).

Table 1: Show the results of difficulty in emotion regulation and suicidal ideation

\begin{tabular}{lllll}
\hline Variable & $\boldsymbol{\beta}$ & SE & T & P \\
\hline
\end{tabular}




\begin{tabular}{lcccc}
\hline Constant & 6.046 & 9.199 & .657 & .514 \\
$\begin{array}{l}\text { Difficulty } \\
\text { in emotion } \\
\text { regulation }\end{array}$ & .214 & .092 & 1.667 & .101 \\
\end{tabular}

Note: $\mathrm{R} 2=.046 ; \mathrm{B}=$ coefficients, $\mathrm{SE} ; \mathrm{Std}$. Error: Dependent variable is suicidal ideation

The 2nd hypothesis results indicated that difficulty in emotion regulation positively predict psychological distress (depression, anxiety and stress) among patients with substance use disorders. Regression coefficients of difficulty in emotion regulation with depression is $(\beta=.322)$, with anxiety is $(\beta=.326)$ and stress $(\beta=.320)$. The result of the second hypothesis seen in (Table $2,3,4$ )

Table 2: Show the results of difficulty in emotion regulation and depression

\begin{tabular}{lcccc}
\hline Variable & B & SE & T & P \\
\hline Constant & .137 & 5.74 & .024 & .981 \\
$\begin{array}{l}\text { Difficulty } \\
\text { in } \\
\text { emotion } \\
\text { regulation }\end{array}$ & .322 & 058 & 2.588 & .012 \\
\hline
\end{tabular}

Note: $\mathrm{R} 2=.104 ; \mathrm{B}=$ coefficients, $\mathrm{SE} ; \mathrm{Std}$. Error: Dependent variable is depression

Table 3: Show the results of difficulty in emotion regulation and anxiety

\begin{tabular}{lcccc}
\hline Variable & B & SE & T & P \\
\hline Constant & .124 & 5.147 & .024 & .981 \\
$\begin{array}{l}\text { Difficulty } \\
\text { in }\end{array}$ & .326 & .052 & 2.622 & .011 \\
$\begin{array}{l}\text { emotion } \\
\text { regulation }\end{array}$ & & & & \\
\hline
\end{tabular}

Note: $\mathrm{R} 2=.106 ; \mathrm{B}=$ coefficients, $\mathrm{SE} ; \mathrm{Std}$. Error: Dependent variable is anxiety

Table 4: Show the results of difficulty in emotion regulation with stress

\begin{tabular}{lcccc}
\hline Variable & $\boldsymbol{\beta}$ & $\mathbf{S E}$ & $\mathbf{T}$ & $\mathbf{P}$ \\
\hline Constant & 2.117 & 5.350 & .0396 & .694 \\
$\begin{array}{l}\text { Difficulty } \\
\text { in emotion } \\
\text { regulation }\end{array}$ & .320 & .054 & 2.569 & .013 \\
\end{tabular}

Note: $\mathrm{R} 2=.102 ; \mathrm{B}=$ coefficients, $\mathrm{SE} ; \mathrm{Std}$. Error: Dependent variable is stress

\section{DISCUSSION}

Results of the present study indicate that the difficulty in emotion regulation positively predicted suicidal ideation and psychological distress. As mentioned in the first hypothesis, difficulty in emotion regulation would positive predicts suicidal ideation among patient with substance use disorders. The finding of the present study also consisted with those studies who have also reported positive correlation in difficulty in emotion regulation and suicidal ideation among patient with substance use disorders.22, 23

Difficulty in emotion regulation is commonly characteristic in a number of clinical settings, and describes several clinically relevant behaviors and problem, such as depression, substance addiction and suicidal ideation.23 Research which are conducted on substance use revealed that the substance intoxication develop a syndrome which directly affects the central nerves system and develop the disturbance in perception, thinking, concentration and decision making which are considered difficulty in emotion regulation. The consequence of the disturbance occur in form of interpersonal relationships problems, guilt feeling inability to inhibit negative behaviors, social isolation feeling loneliness, serious physical and mental health problems suicidal ideation.24 Difficulty in emotion regulation abilities also develop through environmental factors such as changing the location, losing relationship, separation, rejection, isolation and poor coping which increase the risks of anti-social behavior and tendency of suicidal ideation. 25

The second hypothesis of the current study indicated the positive predict the difficulty in emotion regulation with psychological distress (depression, anxiety and stress) among patient with substance use disorders. The finding of the present study also consisted with those studies who have also reported the positive correlation among difficulty in emotion regulation with psychological distress (depression, anxiety and stress) among patient with substance use disorders. The difficulty in emotion regulation is the cause of many addictive behaviors, including, substance abuse, depression, anxiety and stress and other psychological problems. Similarly the difficulties in emotion regulation in adolescents and young adults can lead to depression, anxiety and stress if a person is weak in controlling emotions, they will experience stress and anxiety in stressful circumstances and therefore they will start substance use to control and reduce negative emotions. 26

Difficulty in emotion regulation system itself is a more direct, efficient, and powerful way to override and transform the maladaptive patterns that underlie unhealthy psychological, behavioral, and physiological responses. Positive emotions can play a critical role in breaking the stress cycle by effectively transforming stress at its source.27 Positive emotions have been linked to improved health and increased longevity. They have also been shown in result to increases cognitive flexibility, creativity, receptivity, innovative problem solving, and psychological 
resilience in the face of adversity.28 the literature also suggests that emotional regulation strategies such as positive reappraisal and curtailed rumination exert a protective influence over relapse or exacerbated symptoms in patients with depression, anxiety and stress disorders. 2

\section{CONCLUSION}

The present study demonstrates the link between difficulty in emotion regulation, suicidal ideation and psychological distress, which illustrate that the significant association in these variables among patient with substance use disorders. The findings to the study described that there was a positive association among the difficulty in emotion regulation, suicidal ideation and psychological distress. The views of the current study sample support the hypotheses. The previous studies along with the findings also proved our hypotheses which showed that difficulty in emotion regulation had the high level of suicidal ideation and psychological distress (depression, anxiety and stress). For instances, if an individual had the inability to control their emotions, than they had leads the psychological distress which causes the risky behavior of suicidal ideation.

\section{RECOMMENDATION}

It is recommended that a replication of this study should be conducted with a larger sample. In addition, the inclusion of a control group would be a vital and effective for accurately assess and also the efficacy to the program. It is recommended that the psychologist should work on individuals' difficulty in emotion regulation and coping skill to control suicidal thoughts and attempts and other psychological problems.

\section{REFERENCES}

[1]. Diagnostic and Statistical Manual of Mental Disorders, (1980). American Psychiatric Association,3rd ed.; American Psychiatric Association: Washington, DC, USA.

[2]. Prashant, S., (1991). Drug Abuse and Sociey. New Delhi, Ashish Publishers House, Punjab Bagh. 220.

[3]. Quraishi, S., (2003). Drug Addiction in Pakistan. Book Network Publishers, Karachi. 380p.

[4]. Gelder, M., Mayou, R., Cowen, P.,(2001).Misuse of Alcohol and Drugs.In Shorter Oxford Text book of Psychiatry. 4th edition. Oxford University Press:533-79.

[5]. Cheetham, A., Allen, N. B., Yucel, M., Lubman, D. I., (2010). The role of Affective dysregulation in drug addiction. Clinical Psychology Review, 30, 621-634.

[6]. Bonn, M. O., Vujanovic, A. A., Zvolensky, M. J., (2008). Emotional dysregulation: Association with coping-oriented marijuana use motives among current marijuana users. Substance Use \& Misuse,43(11), 16531665.

[7]. Aguilar, de. F., Verdejo, Garcia. A., Ceverino, A., Montanez, Pareja. M., Lopez, Juarez. E., Sanchez, Barrera. M., Lopez, Jimenez. A., Perez, Garcia. M., (2008). PEPSA Team. Dysregulation of emotional response in current and abstinent heroin users: negative heightening and positive blunting. Psychopharmacology (Berl.). 198:159-166.

[8]. Miller, N. S., \& Giannini, A. J., (1990). The disease model of addiction: a biopsychiatrist.'s view, Journal of Psychoactive Drugs, 22, 83-85.

[9]. Diaz, R. M., Fruhauf, A. G., (1991). The origins and development of self-regulation: A developmental model on the risk for addictive behaviors: $10 \quad$ Beck, A.T., Wright, F. D., Newman, C. F., \& Liese, B. S., (1993). Cognitive therapy of substance abuse. New York: Guilford.

[10].Beck, A. T., (1976). Cognitive therapy and the emotional disorders. New York: International Universities.

[11].Bender, T. W., Gordon, K. H., Bresin, K., \& Joiner, T. E., (2011). Impulsivity and Suicide: The mediating role of painful and provocative experiences. Journal of Affective Disorders, 129, 301-307.

[12].Baumeister, R. F., (1990). Suicide as escape from self. Psychological Review, 97, 90-11.

[13].Mennin, D. S., \& Fresco, D. M., (2009). Emotion regulation as an integrative framework for understanding and treating psychopathology. In A. M. Kring \& D. M. Sloan (Eds.), Emotion regulation in psychopathology: A transdiagnostic approach to etiology and treatment (pp. 356-379). New York:Guilford.

[14].Aldao, A., \& Nolen, S., (2012). When are adaptive strategies most predictive of psychopathology? Journal of Abnormal Psychology, 121, 276-281.

[15].Afifi, T.O., Cox, B.J., Katz, L. Y., (2007). The associations between health risk behaviorsand suicidal ideation and attempts in a nationally representative sample of young adolescents. Canadian Journal of Psychiatry, 52(10), 666-674.

[16]. Anxiety and Depression Association of America (1980) is a U.S. nonprofit organization dedicated to increasing awareness and improving the diagnosis, treatment, and cure of anxiety disorders in children and adults.

[17].SAMHSA (Substance Abuse and Mental Health Services Administration) (2002) Substance use and the risk of suicide among youths. National Household Survey on Drug Abuse Report.

[18].Kelder, S.H., Murray, N.G., Orpinas, P., Prokhorov, A., McReynolds, L., Zhang, Q., \& Roberts, R., (2000). Depression and substance use in minority middle-school students. American Journal of Public Health, 91, 761-766.

[19].Kelly, T. M., Cornelius, J. R., \& Clark, D. B., (2004). Psychiatric disorders and attempted suicide among adolescents with substance use disorders. Drug and Alcohol Dependence, 73(1), 87-97.

[20].Gratz, K. L., \& Roemer, L., (2004). Multidimensional assessment of emotion regulation and dysregulation: Development, factor structure, and initial validation of the Difficulties in Emotion Regulation Scale. Journal of Psychopathology and Behavioral Assessment, 26, 41-54.

[21].Van Spijker, B. A., Batterham, P. J., Calear, A. L., Farrer, L., Christensen, H., Reynolds, J., \& Kerkhof, A., (2014). The Suicidal Ideation Attributes Scale (SIDAS): CommunityBased Validation Study of a New Scale for the Measurement of Suicidal Ideation. Suicide and Life-Threatening Behavior, 44(4), 408-419. DOI: 10.1111/sltb.12084.

[22].Lovibond, P. F., \& Lovibond, S. H., (1995). The structure of negative emotional states: Comparison of the Depression Anxiety Stress Scales (DASS) with the Beck Depression and Anxiety Inventories. Behaviour Research and Therapy, 33(3), 335-343.

[23].Arria, A. M., Grady, K. E., Caldeira, K. M., Vincent, K. B., Wilcox, H. C., \& Wish, E. D., (2009). Suicide ideation among college students: A multivariate analysis. Archives of Suicide Research, 13, 230-246.

[24].Tull, MT., Weiss, N., Adams, C., Gratz, K., (2012). The contribution of emotion regulation difficulties to risky sexual behavior within a sample of patients in residential substance abuse treatment. Addictive Behaviour. 37(10):1084-92. 
[25].Daniel, S. S., \& Goldston, D. B., (2009). Interventions for suicidal youth: a review of the literature and developmental considerations. Suicide and Life-Threatening Behavior, 39(3), 252-268.

[26].Dour, H. J., Cha, C. B., \& Nock, M. K., (2011). Evidence for an emotion cognition interaction in the statistical prediction of suicide attempts. Behavior Research and Therapy, 49, 294298.

[27].Miller, N. S., \& Giannini, A. J., (1990). The disease model of addiction: a biopsychiatrist's view, Journal of Psychoactive Drugs, 22, 83-85.

[28]. Mitchell, L., Johunt., (2013).Emotion dysregulation, ADHD and Smoking. Duke University, durham. Nc; , 09-00211.

[29].Smith, J. M., Alloy, L. B., \& Abramson, L. Y., (2006). Cognitive vulnerability to depression, rumination, hopelessness, and suicidal ideation: Multiple pathways to selfinjurious thinking. Suicide and Life-Threatening Behavior, 36, 443-454.

[30].Fergusson, D. M., Horwood, L. J., \& Swain - Campbell, N., (2002). Cannabis use and psychosocial adjustment in adolescence and young adulthood. Addiction, 97(9), 11231135. 